\title{
A NOTE ON GROUPS OF PRIME POWER EXPONENT SATISFYING AN ENGEL CONGRUENCE
}

\author{
GERALD LOSEY ${ }^{1}$
}

1. In [3] Kostrikin has proved that a Lie algebra of characteristic $p, p$ prime, satisfying the $n$th Engel condition, $n \leqq p$, is locally nilpotent. Using this result he has shown that in a group of exponent $p$ generated by $r$ elements, $r$ finite, one has $G_{m}=G_{m+1}$ (where $G_{i}$ denotes the $i$ th term of the lower central series of $G$ ) and $m$ is dependent only on $p$ and $r$. In this note we shall apply Kostrikin's result to groups of exponent $p^{\alpha}$.

2. Let us denote by $(x, y)$ the group commutator $x^{-1} y^{-1} x y$. We define the symbol $(x, y ; n)$ recursively by

$$
(x, y ; 1)=(x, y), \quad(x, y ; n+1)=((x, y ; n), y),
$$

that is, $(x, y ; n)$ is a left normed commutator of weight $n+1$. A group $G$ is said to satisfy the $n$th Engel congruence if $(x, y ; n) \equiv 1 \bmod G_{n+2}$ for all $x, y \in G$. In a group of exponent $p$ the $(p-1)$ st Engel congruence holds (P. Hall, [1]).

3. If $G=H_{1} \supseteq H_{2} \supseteq H_{3} \supseteq \cdots$ is a descending central series for $G$ having the property that $\left(H_{i}, H_{j}\right) \subseteq H_{i+j}$ for all $i$ and $j$ then one can construct, in the canonical fashion, the Lie ring

$$
L\left(H_{i}\right)=\sum\left(H_{i} / H_{i+1}\right), \quad \text { direct sum }
$$

(cf. Lazard, [4], and Higman, [2]). In particular, if $\left\{G_{i}\right\}$ is the lower central series of $G$ we obtain the Lie ring $L\left(G_{i}\right)$ used by Higman, [2], and Kostrikin, [3]. It has been shown by Higman, [2], that if $G$ satisfies the $n$th Engel congruence then the relation $n ! x y^{n}=0$ is satisfied for all $x, y \in L\left(G_{i}\right)$.

4. Since $G_{i} \subseteq H_{i}$ for all $i$, we have $G_{i} H_{i+1} / H_{i+1}$ a subgroup of $H_{i} / H_{i+1}$. Hence

$$
L^{*}\left(H_{i}\right)=\sum\left(G_{i} H_{i+1} / H_{i+1}\right), \quad \text { direct sum, }
$$

is a subgroup of $L\left(H_{i}\right)$. For $x \in G_{i}$ and $y \in G_{j}$ we have $(x, y) \in G_{i+j}$ $\subseteq G_{i+j} H_{i+j+1}$ and so it follows that $L^{*}\left(H_{i}\right)$ is a Lie subring of $L\left(H_{i}\right)$. Moreover, the mappings $\phi_{i}: G_{i} / G_{i+1} \rightarrow G_{i} H_{i+1} / H_{i+1}$ given by $x G_{i+1}$ $\rightarrow x H_{i+1}$ are homomorphisms. The mapping $\phi=\sum \phi_{i}$ is then easily

Received by the editors February 5, 1963.

1 This research was supported by the National Science Foundation under contract G-19052. 
seen to be a homogeneous Lie ring homomorphism of $L\left(G_{i}\right)$ onto $L^{*}\left(H_{i}\right)$. Thus $L^{*}\left(H_{i}\right)$ is a subring of $L\left(H_{i}\right)$ and a homomorphic image of $L\left(G_{i}\right)$.

5. We can now state and prove our result.

Theorem. Let $G$ be a finitely generated group of exponent $p^{\alpha}, p$ prime, such that

(i) G satisfies the $(p-1)$ st Engel congruence,

(ii) for some integer $m>0, G_{m}$ has exponent $p$.

Then there exists an integer $n$ such that $G_{n}=G_{n+1}$.

Proof. In [5] Zassenhaus shows that the sequence of subgroups $H_{k}=\prod G_{i}^{p^{j}}$, the product taken over all $i$ and $j$ such that $i p^{j} \geqq k$, form a descending central series for $G$ satisfying the conditions $\left(H_{r}, H_{s}\right)$ $\subseteq H_{r+s}$ and $H_{r}^{p} \subseteq H_{r p} \subseteq H_{p+1}$. Hence $L\left(H_{i}\right)$ is a Lie algebra of characteristic $p$ and, hence, so is $L^{*}\left(H_{i}\right)$. Since the $(p-1)$ st Engel congruence holds in $G$, the relation $(p-1) ! x y^{p-1}=0$ holds in $L\left(G_{i}\right)$ and hence also in the homomorphic image $L^{*}\left(H_{i}\right)$. Since $L^{*}\left(H_{i}\right)$ has characteristic $p$, this implies that the $(p-1)$ st Engel condition, $x y^{p-1}=0$, holds in $L^{*}\left(H_{i}\right)$. Since $G$ is finitely generated, $L^{*}\left(H_{i}\right)$ is also finitely generated and so, by Kostrikin's result, $L^{*}\left(H_{i}\right)$ is nilpotent. Thus for some integer $N$ we have $G_{n} \subseteq H_{n+1}$ or

$$
G_{n} \subseteq G_{n+1} G_{n}^{p} G_{n-1}^{p} \cdots=\left\lfloor G_{i}^{p^{i}}\right.
$$

for all $n \geqq N$. By taking $n$ large enough we can ensure that in (1) each term $G_{k}$ with $k<m$ occurs with exponent $\geqq p^{\alpha}$. Then (1) will reduce to $G_{n} \subseteq G_{n+1}$, that is, $G_{n}=G_{n+1}$.

6. This result is not as strong as one might hope for. We would like to have the index $n$ depend only on $p^{\alpha}, m$ and the number of generators of $G$. However it is not possible to assert the existence of a universal group $G$ generated by $r$ elements and satisfying the conditions of the theorem and having any other such group as a homomorphic image. Since the sequence $\left\{H_{i}\right\}$ of Zassenhaus is the most rapidly descending central series such that $H_{i} / H_{i+1}$ has exponent $p$ it seems unlikely that a stronger result can be obtained using the method of this note unless one can strengthen Kostrikin's theorem.

\section{REFERENCES}

1. P. Hall, $A$ contribution to the theory of groups of prime power order, Proc. London Math. Soc. 36 (1933), 29-95. 
2. G. Higman, On finite groups of exponent five, Proc. Cambridge Philos. Soc. 52 (1956), 381-390.

3. A. I. Kostrikin, On Burnside's problem, Dokl. Akad. Nauk SSSR 119 (1958), 1081-1084. (Russian)

4. M. Lazard, Sur les groupes nilpotents et les anneaux de Lie, Ann. Ecole Norm. Sup. (3) 71 (1954), 101-190.

5. H. Zassenhaus, Ein Verfahren, jeder endlichen p-Gruppe eine Lie-Ring mit der Charakteristik p zuzuordnen, Abh. Math. Sem. Hamburg Univ. 13 (1939), 200-207.

UNIVERSITY OF WisCONSIN

\section{A GENERALIZATION OF THE CARTAN- BRAUER-HUA THEOREM}

CHARLES J. STUTH

Let $K$ be a division ring. Then $K^{\prime}$ will denote the multiplicative group of $K$. If $S$ is a subset of $K$, then $S^{*}$ will denote the division ring generated by $S$, and $C(S)$ will denote the centralizer of $S$ in $K$. If $G$ is a subgroup or a subdivision ring contained in $K$, then $Z(G)$ is the center of $G$. If $x$ and $y$ are elements of $K^{\prime}$, then $(x, y)=x y x^{-1} y^{-1}$ and $x^{y}=y x y^{-1}$. If $b$ is an element of a group $A$, then $\operatorname{Cl}(A, b)$ will denote the group which is generated by the conjugate class of $b$ in $A$.

A set is central in $K$ if each of its elements is in $Z(K)$. A group $G$ is $n$-subnormal in a group $H$ if there are groups $G_{1}, \cdots, G_{n-1}$ such that $G=G_{n} \triangleleft G_{n-1} \triangleleft \cdots \triangleleft G_{1} \triangleleft G_{0}=H$. A group $G$ is subnormal in a group $H$ if $G$ is an $n$-subnormal subgroup of $H$ for some $n$. A group $G$ is invariant under a group $H$ if $g^{h} \in G$ for all $g \in G$, all $h \in H$. A noncentral subgroup $G$ is of type $I$ if $G$ is invariant under a noncentral subnormal subgroup of $K^{\prime} . K$ has the property $\mathrm{P}_{n}$ if for every subdivision ring $H$ of $K$ such that $H^{\prime}$ is invariant under a noncentral $n$ subnormal subgroup of $K^{\prime}$ it follows that $H$ is central or $H=K$.

The Cartan-Brauer-Hua Theorem [2] states that a division ring has property $\mathrm{P}_{0}$. Herstein and Scott [1] generalized this to $\mathrm{P}_{1}$. Schenkman and Scott [5] extended the Cartan-Brauer-Hua Theorem by showing that a division ring has property $\mathrm{P}_{n}$ for all $n$ if each of its subdivision rings which is invariant under a subnormal subgroup is normal in some subnormal subgroup of the division ring.

Theorem 1 of this paper shows that a division ring has property $\mathrm{P}_{n}$ for all $n$. Then results are developed from this concerning the subnormal subgroups of $K^{\prime}$ and more generally for the subgroups of type $I$ in $K^{\prime}$.

Received by the editors January 16, 1963. 